\title{
High-resolution spectroscopic observations of the peculiar planetary nebula Me $1-1^{\star}$
}

\author{
C. B. Pereira ${ }^{1}$, L. F. Miranda ${ }^{2}$, V. V. Smith ${ }^{3}$, and K. Cunha ${ }^{3, \star \star}$ \\ 1 Observatório Nacional-MCT, Rua José Cristino, 77, CEP 20921-400 São Cristóvão, Rio de Janeiro-RJ, Brazil \\ e-mail: claudio@on.br \\ 2 Instituto de Astrofísica de Andalucía, CSIC, Apdo. 3004, 18080 Granada, Spain \\ e-mail: lfm@iaa.es \\ 3 NOAO, Casilla 603, La Serena, Chile \\ e-mail: [smith; cunha]@noao.edu
}

Received 31 July 2007 / Accepted 18 October 2007

\section{ABSTRACT}

\begin{abstract}
Aims. We determined the atmospheric parameters and evolutionary state of the cool stellar component of the peculiar planetary nebula Me 1-1 and analyzed its spatio-kinematic structure.

Methods. High-resolution optical spectroscopy was used in an abundance analysis of the cool component in Me 1-1. Narrow-band images were used to study the nebula.

Results. The stellar parameters derived for the cool star in Me 1-1 are $T_{\text {eff }}=4500 \mathrm{~K}$ and $\log g=2.0$, which correspond to a spectral type of K(1-2) II. This star is rapidly rotating with a projected rotational velocity of $V_{\text {rot }}=90 \mathrm{~km} \mathrm{~s}^{-1}$. Its estimated mass is $3.5 M_{\odot}$ with a distance of $6 \mathrm{kpc}$ and a luminosity of $370 L_{\odot}$. The cool star in Me 1-1 has nearly solar iron and calcium abundances. Barium, a monitor of s-process nucleosynthesis, is not enriched. We show that this cool star has reached the base of the RGB and its accreted matter has been diluted by the deepening convective envelope. The images strongly suggest that Me 1-1 is an edge-on ring with incipient bipolar lobes. The central star is displaced $\sim 400 \mathrm{AU}$ from the center of the nebula. The expansion velocity of the ring ranges from 10 and $13 \mathrm{~km} \mathrm{~s}^{-1}$ in the different emission lines. Broad $\left(\simeq 600 \mathrm{~km} \mathrm{~s}^{-1}\right)$ wings are detected in the $\mathrm{H} \alpha$ emission, probably with a stellar origin. A comparison of Me 1-1 with other ring-like planetary nebula shows that most of them host off-center binary central
\end{abstract} stars.

Key words. ISM: planetary nebulae: individual: Me 1-1 - stars: binaries: general - stars: late-type - stars: abundances

\section{Introduction}

The Strasbourg-ESO Catalogue of Planetary Nebulae reports that 1820 objects have been classified at least once as planetary nebulae (PNe; Acker et al. 1992). However, there are only a few PNe known to contain close binary nuclei. Bond (2000) lists 16 objects, which he divides into three classes: those that behave as eclipsing binaries and/or show heating effects into the atmosphere of a main sequence star; those that behave as spectroscopic binaries; and, finally, those that are classified as "Abell 35-type" objects. The "Abell 35-type" objects represent what has been previously classified as "peculiar PNe" according to Lutz (1977). In particular, Lutz (1977) called peculiar PNe, those objects that have nebular emission lines in combination with an absorption spectrum of a cool central star (of spectral types A to K). Since the cool star is not hot enough to account for the ionization of the nebulae, these peculiar PNe should be binary systems consisting of a faint hot star (responsible for the ionization of the nebulae) plus a bright cool star that is the source of the absorption spectrum. Nowadays, it is known that some of the

* Based on observations collected at the Centro Astronómico Hispano Alemán (CAHA) at Calar Alto, operated jointly by the MaxPlanck Institut für Astronomie and the Instituto de Astrofísica de Andalucía (CSIC).

$\star \star$ On leave from Observatorio Nacional/MCT, Rio de Janeiro Brazil. objects in Lutz's (1977) list belong to a small subsample of symbiotic stars called yellow symbiotics (Schmid \& Nussbaumer 1993).

The cool central stars of peculiar PNe are interesting to study because mass-transfer may have happened from the former AGB star, which is now the hot star in the binary system. Another interesting point to investigate is the rotational velocities of the cool central stars. As discussed by Jeffries \& Stevens (1996) there is a class of wide binaries containing hot white dwarfs and rapidly rotating magnetically active cool stars. In the mechanism proposed by these authors the rapid rotation of the cool star is probably due to spin-up from the accretion of material from a massive AGB wind. In fact, some peculiar PNe with such spectroscopic characteristics, such as Abell 35 and LoTr 5 , have already been reported in the literature (Thévenin \& Jasniewicz 1997). In addition, Smith et al. (2001) and Pereira et al. (2005) show that the cool stellar components of the $\mathrm{D}^{\prime}$-type symbiotics S 190, HD 330036 and AS 201 also behave like those in Abell 35 and LoTr 5. In particular, these stars are rapid rotators that show enhanced abundances of carbon and heavy elements produced by s-process nucleosynthesis.

Recently, Shen et al. (2004) have used low-resolution spectroscopy to detect a cool stellar component at the center of the PN Me 1-1 (Hen 2-441). Therefore, Me 1-1 can be included in this family of peculiar PNe. Motivated by the above discussion, 
in this paper we investigate via high-resolution spectroscopy whether Me 1-1 behaves like the other peculiar PNe and $\mathrm{D}^{\prime}$-type symbiotics analyzed so far. In particular, our goal is to investigate whether the cool star of Me 1-1 is a rapid rotator and if it shows enhanced abundances of heavy elements produced by s-process nucleosynthesis. In addition, high resolution spectroscopy is useful in investigating the internal kinematics of the nebulae.

\section{Observations}

Spectroscopic observations were obtained using the FOCES spectrograph (Pfeiffer et al. 1998) with the $2.2 \mathrm{~m}$ telescope at the Calar Alto Observatory (Almería, Spain) on August 1, 2006. The detector was a Site\#1d CCD with 2168 pixels in the spectral direction. The fibre of the spectrograph was combined with a diaphragm of $2.5^{\prime \prime}$ centered on the central star and covering the central regions of the nebula. A slit width of $200 \mu \mathrm{m}$ was used resulting in a resolving power of $R=40000$ and wavelength coverage from $\approx 4750 \AA$ to $9600 \AA$. The nominal $\mathrm{S} / \mathrm{N}$ ratio of the observed spectra was evaluated by measuring the rms flux fluctuation in selected continuum windows, and typical values were $S / N \sim 70-90$ for $2 \times 3600$ s of integration time. The spectra were reduced using the IRAF data package and the data reduction consisted of the following standard steps: CCD bias correction, flatfielding, spectrum extraction and wavelength calibration with a ThAr lamp. No absolute flux calibration was obtained.

In order to complement the analysis of Me 1-1, we have used images from the IAC Catalog of Northern Galactic Planetary Nebulae (Manchado et al. 1996). These images (kindly provided by Guerrero) have been obtained with the Nordic Optical Telescope at Roque de los Muchachos Observatory (La Palma, Spain) in three narrow-band filters that isolate the light of the $\mathrm{H} \alpha,[\mathrm{N}$ II $] 6584$ and [O III]5007 emission lines. The spatial scale and spatial resolution of the images are $0.139^{\prime \prime} \mathrm{pixel}^{-1}$ and $\simeq 0.7^{\prime \prime}$, respectively. More details about the observations can be found in Manchado et al. (1996).

\section{Analysis and results}

\subsection{Determination of the atmospheric parameters and abundances}

The high resolution absorption spectrum of Me 1-1 shows the presence of large numbers of absorption features broadened by a large rotational velocity, similar to what has been previously observed for other $\mathrm{D}^{\prime}$-type symbiotics. The top panels of Figs. 1 and 2 show the observed Calar Alto spectra of Me 1-1 in the spectral regions around $6150 \AA$ and $6440 \AA$, respectively. The region around $6150 \AA$ contains Fe I, Fe II and $\mathrm{Ba}$ II lines while the region around $6440 \AA$ contains Fe I and Ca I lines. In the bottom panels of Figs. 1 and 2 we also show the rotationally broadened spectra of the $\mathrm{D}^{\prime}$-type symbiotic S 190 taken with FEROS spectrograph at ESO, for comparison.

Due to rapid rotation, the spectral lines in Me 1-1 become blended and this prevents a photospheric analysis based on traditional methods of measuring the equivalent widths of individual lines. Thus in order to do an abundance analysis it is necessary to carefully select spectral regions that contain broadened features whose respective strengths are approximately dominated by single species. In particular, the spectral region near $6150 \AA$ (Fig. 1) was selected as it contains a number of broadened features whose respective strengths are dominated by single species

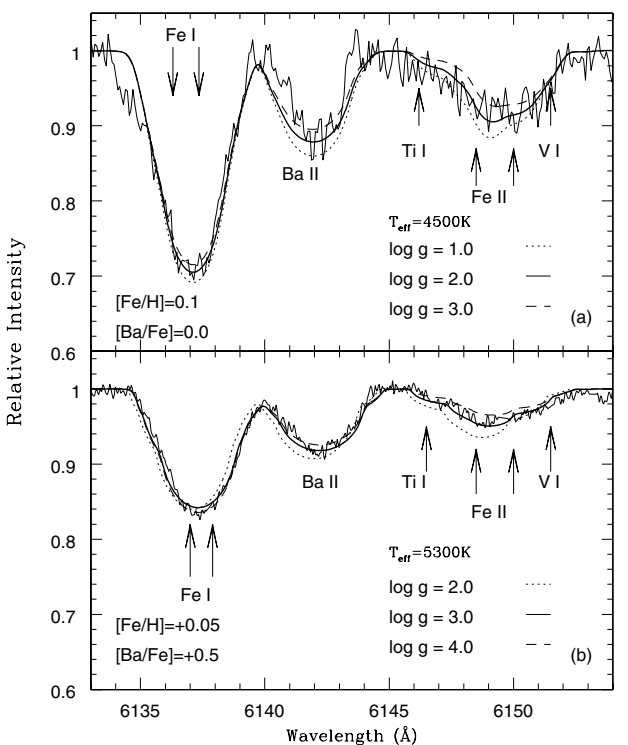

Fig. 1. Observed and synthetic spectra for Me 1-1 a) and S 190 b) displaying broad lines and indicating rotationally broadened profiles. The observed spectra are plotted along with three synthetic spectra computed for three different surface gravities showing the sensitivity of the Fe II (and Ba II to $\log g$ ).

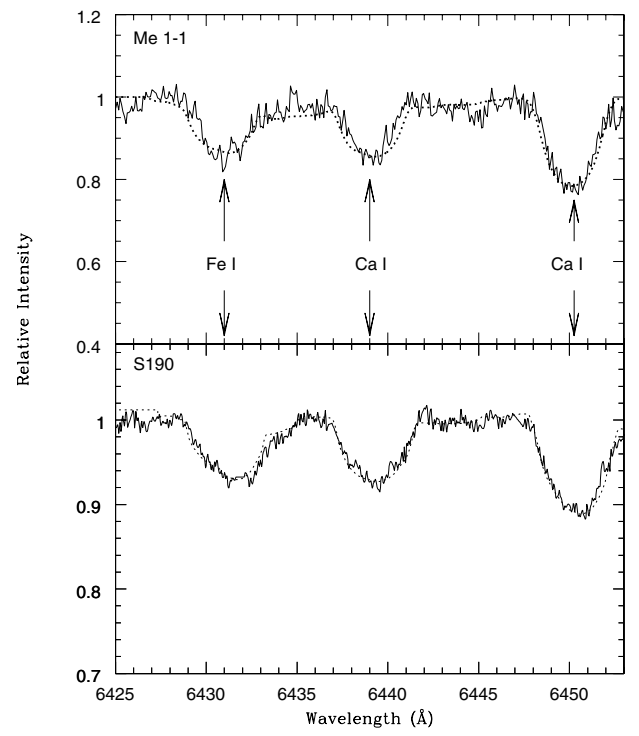

Fig. 2. Observed and synthetic spectra for Me 1-1 (top panel) and S 190 (bottom panel) in the spectral regions 6425-6453 $\AA$. The solid lines in each panel correspond to the observed spectra and dotted lines correspond to the synthetic spectra calculated for $\log \epsilon(\mathrm{Ca})=6.33$ using $T_{\text {eff }}=4500 \mathrm{~K}$ and $\log g=2.0$ for Me $1-1$ and $T_{\text {eff }}=5300 \mathrm{~K}$ and $\log g=3.0$ for $\mathrm{S} 190$.

of Fe I, Fe II (as well as Ba II). We note that unfortunately only a few isolated Fe II lines are found in spectra of rapidly rotating cool stars such as the one studied here. In this paper, we also analyzed one Ba II feature whose abundance serves as a monitor of s-process nucleosynthesis.

Synthetic spectra were calculated using the MOOG LTE synthesis code (Sneden 1973) together with a Kurucz \& Bell (1995) linelist and Kurucz ATLAS9 model atmospheres. We computed a grid of synthetic spectra for model atmospheres covering a range in effective temperature ( $T_{\text {eff }}$ from 4500 to $7000 \mathrm{~K}$ ), surface gravity ( $\log g$; from 0.0 to 4.0 ), microturbulent velocity ( $\xi$; 
Table 1. Atmospheric parameters for the cool central star in Me 1-1.

\begin{tabular}{l|l}
\hline \hline$T_{\text {eff }}$ & $4500 \pm 100 \mathrm{~K}$ \\
$\log g$ & $2.0 \pm 0.5 \mathrm{dex}$ \\
{$[\mathrm{Fe} / \mathrm{H}]$} & $0.10 \pm 0.3 \mathrm{dex}$ \\
$\xi$ & $2.5 \pm 0.5 \mathrm{~km} \mathrm{~s}^{-1}$ \\
$V_{\text {rot }}$ & $90 \pm 10 \mathrm{~km} \mathrm{~s}^{-1}$ \\
\hline
\end{tabular}

from 1.0 to $4.0 \mathrm{~km} \mathrm{~s}^{-1}$ ), and five values of metallicity ([A/H]; $0.0,-0.5,-1.0,-1.5$ and -2.0$)$. Each of these synthetic spectra was compared to the observed spectrum in the interval between 6133-6152 $\AA$ (Fig. 1). Our derivation of the effective temperature and surface gravity for the studied cool star is based upon the analysis Fe I and Fe II lines. It is worth noting that these behave as "single" spectral lines, since the whole absorption profile is dominated by them.

More precisely, our determination of the atmospheric parameters relied on fits to observed and synthetic spectra of two Fe I lines (at $6136.62 \AA$ and $6137.69 \AA$ ) and two Fe II lines (at $6147.74 \AA$ and $6149.26 \AA$ ). We adopted in this study basically the same approach as the one discussed in our previous study (Smith et al. 2001). Table 1 provides the final adopted atmospheric parameters for the cool star in Me 1-1. We note that $T_{\text {eff }}$ and $\log g$ were also re-derived here for the comparison star S 190 and these are in agreement, as expected, with those obtained previously by Smith et al. (2001).

For each synthetic spectrum, corresponding to given values of $T_{\text {eff }}, \log g, \xi,[\mathrm{A} / \mathrm{H}]$, we first determined the abundance of Fe I and the projected rotational velocity $(v \sin i)$ that best reproduce the observed profile of the two Fe I lines mentioned above. This is done by searching for the values of the Fe abundance and $v \sin i$ that minimize the merit function $\chi^{2}=\sum\left(f_{\text {synthetic }}-f_{\text {observed }}\right)^{2}$ ( $f$ represents the normalized spectral flux around the selected lines). Then, we repeated the procedure to determine the abundance of $\mathrm{Fe}$ and $v \sin i$ that reproduce the profile of the two Fe II lines mentioned above. In this way, we derive the best fit values of the Fe abundance and $v \sin i$ for each set of $T_{\text {eff }}, \log g$, $\xi$, $[\mathrm{A} / \mathrm{H}]$. The atmospheric parameters are then determined as those values of $T_{\text {eff }}, \log g, \xi$ and $[\mathrm{A} / \mathrm{H}]$ for which the abundance derived from Fe I equals the abundance of Fe II at the same projected rotational velocity.

The estimated $1 \sigma$ uncertainties in the atmospheric parameters are related to the resolution of the grid of synthetic spectra used, and indicate that $T_{\text {eff }}$ and $\log g$ are constrained to about $\pm 100 \mathrm{~K}$ and \pm 0.5 , respectively. The uncertainty in rotational velocity comes from the fit procedure and is about $\pm 10 \mathrm{~km} \mathrm{~s}^{-1}$.

Figure 1 shows three synthetic spectra and the observed data for Me 1-1 (top panel), as well as that of S 190 (bottom panel), which is shown for comparison. The profiles represented by solid lines in each panel correspond to the synthetic spectra which were calculated with the adopted stellar parameters in Table 1. The best fit synthesis was obtained for iron and barium abundances roughly solar. The dotted and dashed lines represent synthetic spectra for $\log g$ 's differing by \pm 1.0 dex. Note that smaller surface gravities increase the line strength and this effect for the Fe II lines is used in order to estimate $\log g$. Best fits to the observed spectra indicate that the rotational velocity for the cool star in Me 1-1 is $V \sin i=90 \pm 10 \mathrm{~km} \mathrm{~s}^{-1}$. The large projected rotational velocity found for our target is similar to what has been previously obtained for the other $\mathrm{D}^{\prime}$-type symbiotic stars S 190 , HD 330036, and AS 201 (Smith et al. 2001; Pereira et al. 2005; $v \sin i=100 \pm 10,100 \pm 10$ and $25 \pm 10 \mathrm{~km} \mathrm{~s}^{-1}$, respectively).

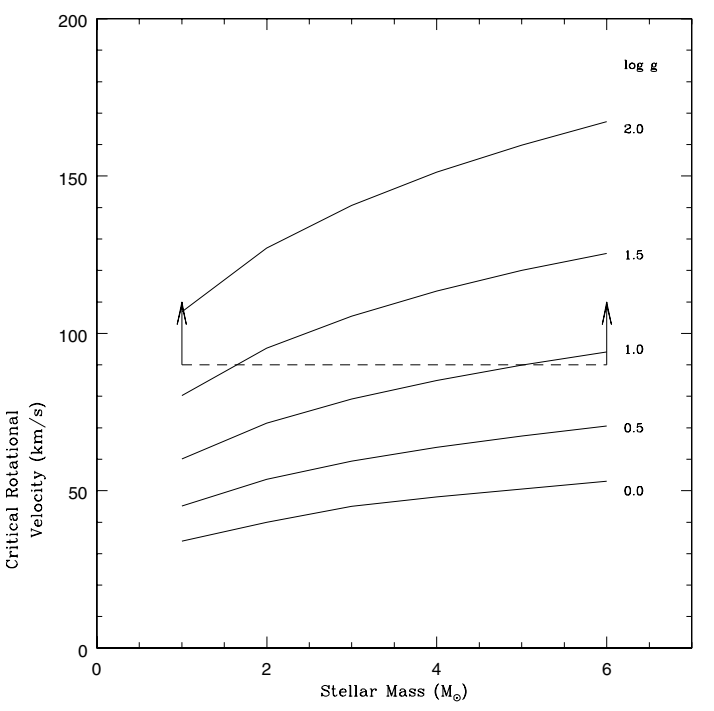

Fig. 3. The critical breakup velocity as a function of stellar mass for different values of $\log g$ from 0.0 to 2.0 (solid lines). The dashed horizontal line with arrows shows v sin i for the cool central star in Me 1-1 $\left(90 \mathrm{~km} \mathrm{~s}^{-1}\right)$. The arrows indicate that this value is a lower limit to the true equatorial rotational velocity.

In the top panel of Fig. 2 we show the synthetic spectrum for the cool star in Me 1-1 in the spectral region around $6440 \AA$, where $\mathrm{Fe} I$ and $\mathrm{CaI}$ lines are observed. The synthetic spectra were calculated for the adopted stellar parameters which are presented in Table 1 . The agreement between the observed and synthetic profiles in this region confirms the atmospheric parameters obtained from the $6150 \AA$ region and, in addition, it indicates that the $\mathrm{Ca}$ abundance is also nearly solar. Finally, the derived values of $T_{\text {eff }}$ and $\log g$ from this high-resolution study of the cool component in Me 1-1 suggest that it is a $\mathrm{K}(1-2) \mathrm{II}$ spectral type (Schmidt-Kaler 1982) that is comparable to the $\mathrm{K}(3-4) \mathrm{II}$ type given by Shen et al. (2004).

\subsection{The rotation of the Me 1-1}

The critical breakup velocity as a function of stellar mass and surface gravity can be written as:

$v_{\text {crit }}=\sqrt{\frac{G M_{\star}}{R_{\star}}}=34\left(g M_{\star}\right)^{1 / 4}$

where $v_{\text {crit }}$ is in $\mathrm{km} \mathrm{s}^{-1}, M_{\star}$ is in units of $M_{\odot}$ and $g$ is the surface gravity in $\mathrm{cm} \mathrm{s}^{-2}$. Figure 3 shows the curves $v_{\text {crit }}$ versus $M_{\star}$ for several constant values of $\log g$. The dashed horizontal line in Fig. 3 corresponds to the derived projected rotational velocity (see above). Figure 3 shows that for any stellar mass, the surface gravity of the cool central star in Me 1-1 must be larger than $\log g \approx 1.5$ in the sense that its rotational velocity never exceeds the critical velocity. The spectroscopically derived value of $\log g=2.0$ does not violate this minimum $\log g$.

\subsection{Emission lines: profiles and kinematics}

The (nebular) emission lines of Me 1-1 between $3530 \AA$ and $7420 \AA$ have been identified by Shen et al. (2004). Beyond $7500 \AA$, we also identify in our spectrum several Paschen emission lines, as well as the [Ar III] $\lambda 7751,[\mathrm{Cl}$ IV $] \lambda \lambda 7530,8046$ and [S III] $] \lambda$ 9069,9531 emission lines. 

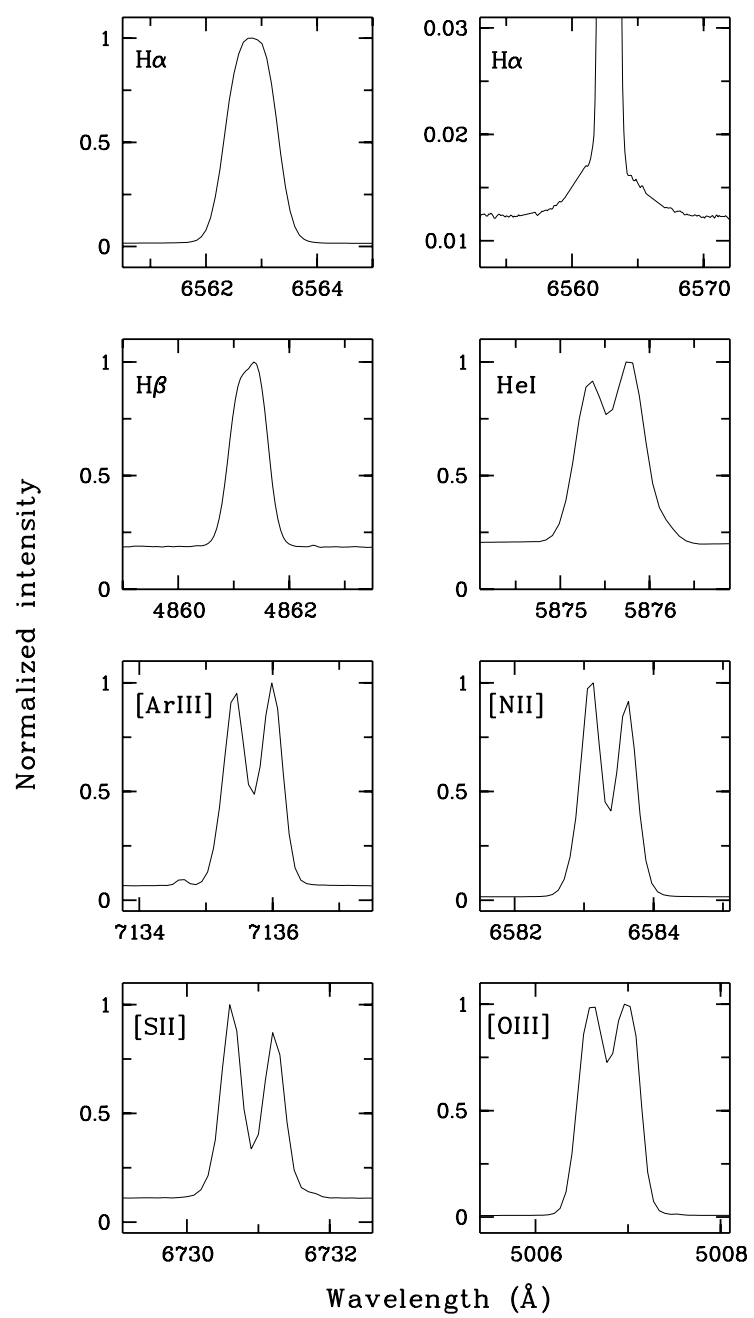

Fig. 4. Line profiles of several emission lines in Me 1-1. The intensity of each line has been normalized to its intensity peak. The $\mathrm{H} \alpha$ profile is presented at two different scales in order to show the broad wings of the $\mathrm{H} \alpha$ emission.

Figure 4 shows the line profiles of several emission lines. All metal lines in our spectrum present a double-peaked profile. Surprinsingly, the velocity separation between the peaks presents a very narrow range of values between $20-21 \mathrm{~km} \mathrm{~s}^{-1}$ for the $\mathrm{He} \mathrm{I}$ and $[\mathrm{Cl} \mathrm{IV}]$ lines and $27 \mathrm{~km} \mathrm{~s}^{-1}$ for the [S II] lines. For the [N I], [N II], [O I], [O III], [Ar III] and [S III] emission lines the velocity separation is $22-25 \mathrm{~km} \mathrm{~s}^{-1}$ without any trend to higher velocity separation with lower ionization potential, as it is usually observed in PNe. The velocity separation between the peaks indicates expansion velocities between 10 and $13 \mathrm{~km} \mathrm{~s}^{-1}$.

The nebular $\mathrm{H} \alpha$ and $\mathrm{H} \beta$ emission lines do not show a doublepeaked profile (Fig. 4). However, the $\mathrm{H} \alpha$ profile cannot be fitted with a single gaussian line profile and $\mathrm{H} \beta$ shows a shoulder that suggests the presence of two velocity components. Probably, the lack of double-peaked profiles is due to the large thermal width in the hydrogen lines. In addition, broad wings are detected in $\mathrm{H} \alpha$ and $\mathrm{H} \beta$ extending $\simeq 600 \mathrm{~km} \mathrm{~s}^{-1}$ and $\simeq 400 \mathrm{~km} \mathrm{~s}^{-1}$, respectively. These broad wings probably have a stellar origin. We note that the velocity centroid of the nebular $\mathrm{H} \alpha$ emission seems to be blueshifted by $7.5 \pm 1.8 \mathrm{~km} \mathrm{~s}^{-1}$ with respect to the velocity centroid of the broad wings. Given the different origins of the narrow (nebular) and the broad (stellar) $\mathrm{H} \alpha$ emission components, the velocity difference could be related to orbital motion of the central star (see Miranda et al. 2001). New high-resolution

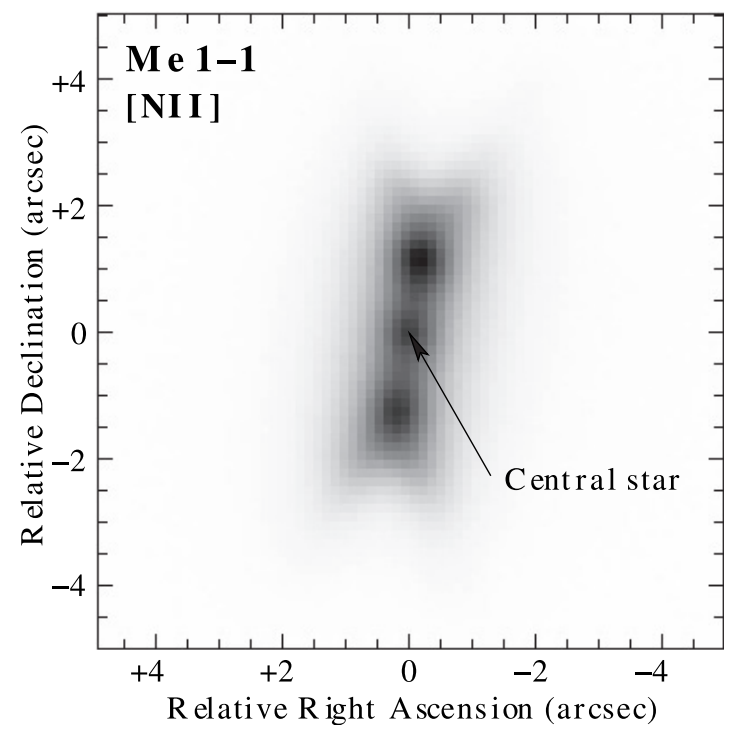

Fig. 5. Grey-scale representation of the $[\mathrm{N}$ II] image of Me 1-1. The grey leves are linear. The origin $(0,0)$ corresponds to the position of the central star (labelled).

spectra would be very valuable to study the $\mathrm{H} \alpha$ line profile in more detail.

Shen et al. (2004) tentatively identified the Raman scattering feature at $6825 \AA$. In our spectrum this feature is not present. We have also checked for the possible presence of the Raman features at $4850 \AA, 6545 \AA$ and $7088 \AA$ but they could not be identified in our spectrum.

\subsection{Direct images}

Figure 5 shows a grey-scale representation of the $[\mathrm{N} \mathrm{II}] \mathrm{im}-$ age of Me 1-1 (the $\mathrm{H} \alpha$ and [O III] images are not show here). Me 1-1 consists of three bright knots, a central one corresponding to the central star, and two outer ones. The three knots are aligned along $\mathrm{PA} \simeq 352^{\circ}$. Faint emission with a "V" (or inverted "V") shape emanates from the outer knots. The separation between the outer knots amounts $\simeq 1.9^{\prime \prime}$ in $\mathrm{H} \alpha$ and [O III] and $\simeq 2.3^{\prime \prime}$ in $[\mathrm{N}$ II] . A visual inspection of the [N II] and $\mathrm{H} \alpha$ images shows that the central star is not located at the middle of the outer knots but displaced towards PA $352^{\circ}$ (Fig. 1; see also Manchado et al. 1996). Given the brightness and compactness of the outer knots (and central star), their relative positions (defined by their centroid) can be determined with high accuracy. We have used different methods (one- and two-dimensional gaussian and paraboloid line fits) to determinate the centroids, and both the $\mathrm{H} \alpha$ and $[\mathrm{N}$ II] images are consistent with a displacement of $0.067^{\prime \prime} \pm 0.010^{\prime \prime}$ towards PA $352^{\circ}$.

\section{Discussion}

\subsection{Distance and evolutionary state}

The stellar parameters derived here for the cool star in Me 1-1 are found in Table 1. These parameters can be used to locate the star on a $\log g-\log T_{\text {eff }}$ diagram so that its position can be compared to stellar evolutionary model tracks. Figure 6 shows the location of cool star of Me 1-1 as well as that of three yellow $\mathrm{D}^{\prime}$-type symbiotic stars (Smith et al. 2001; Pereira et al. 2005) plus model tracks computed by Schaerer et al. (1993) for solar 


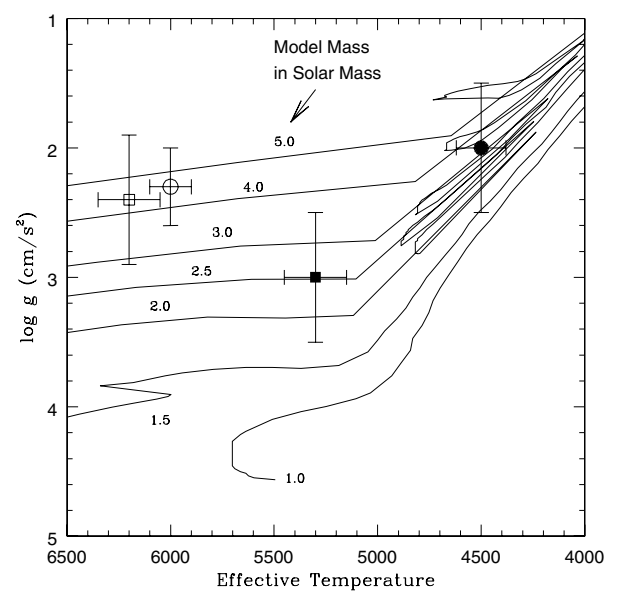

Fig. 6. The position of Me 1-1 (filled circle) in a $\log g-\log T_{\text {eff }}$ diagram along with stellar model evolutionary tracks from Schaerer et al. (1993). The positions of three $\mathrm{D}^{\prime}$-type symbiotic stars is also shown, S 190 (filled square), AS 201 (open circle) and HD 330036 (open square).

metallicity. The stellar parameters derived for the cool star of Me 1-1 place it on the giant branch with a mass of $\sim 3.5 \pm 2.0 M_{\odot}$.

The relation among temperature, gravity and mass, and the $V$ magnitude and the interstellar absorption $\left(A_{V}\right)$ is given by:

$$
\begin{aligned}
\log r(\mathrm{kpc})=\frac{1}{2} & \left(\log \frac{M_{\star}}{M_{\odot}}+0.4\left(V-A_{\mathrm{V}}\right)\right. \\
& \left.+4 \log T_{\text {eff }}-\log g-16.5\right) .
\end{aligned}
$$

Adopting $T_{\text {eff }}=4500 \mathrm{~K}$ and $\log g=2.0$, Eq. (2), can be written as

$\log r(\mathrm{kpc})=\frac{1}{2}\left(\log \frac{M_{\star}}{M_{\odot}}+0.993\right)$

where we took the $V$ magnitude and interstellar absorption values from Shen et al. (2004; $\left.\left(V-A_{V}\right)=12.2\right)$ and assumed $M_{\text {bol } \odot}=+4.74$ (Bessel 1998). For $M_{\star}=3.5 M_{\odot}$ (Fig. 6), we obtain a distance $r \simeq 6 \pm 4 \mathrm{kpc}$. Previous distance determinations for Me 1-1 found $\simeq 4.6 \mathrm{kpc}$ (Cahn et al. 1992; Phillips 2004 ) and $\simeq 8 \mathrm{kpc}$ (Shen et al. 2004). At $6 \mathrm{kpc}$, the luminosity of the cool star in Me $1-1$ is $370 L_{\odot}$.

\subsection{Me 1-1 in comparison with $D^{\prime}$-type symbiotic stars}

Although Figs. 1 and 2 show that the absorption spectrum of Me 1-1 is similar to that of the $\mathrm{D}^{\prime}$-type symbiotic star S 190, from our spectroscopic analysis we did not find any barium enrichment. In fact, Shen et al. (2004) also failed to detect any barium line at $4554 \AA, 5854 \AA$ and at $6142 \AA$. Had Me 1-1 been barium-enriched, some of these Ba lines would not have escaped detection even in a low resolution spectrum.

The abundance analyses by Smith et al. (2001) and Pereira et al. (2005) of other three $\mathrm{D}^{\prime}$-symbiotics (S190, HD 330036, and AS201) have all found [Ba/Fe] enhancements of $\sim+0.5-0.9$ dex, thus the lack of a similar enrichment in Me 11 seems to be puzzling. A closer inspection of the evolutionary states of the three $\mathrm{D}^{\prime}$-symbiotics, however, may reveal the reason why Me 1-1 does not exhibit a measurably elevated $\mathrm{Ba} / \mathrm{Fe}$ abundance ratio. Figure 7 illustrates the derived values of $[\mathrm{Ba} / \mathrm{Fe}]$ as a function of the effective temperatures for these $\mathrm{D}^{\prime}$-symbiotics. This figure reveals a significant decline in $[\mathrm{Ba} / \mathrm{Fe}]$ as $T_{\text {eff }}$ decreases. Reinspection of Fig. 7 shows that the $\mathrm{D}^{\prime}$-symbiotic stars

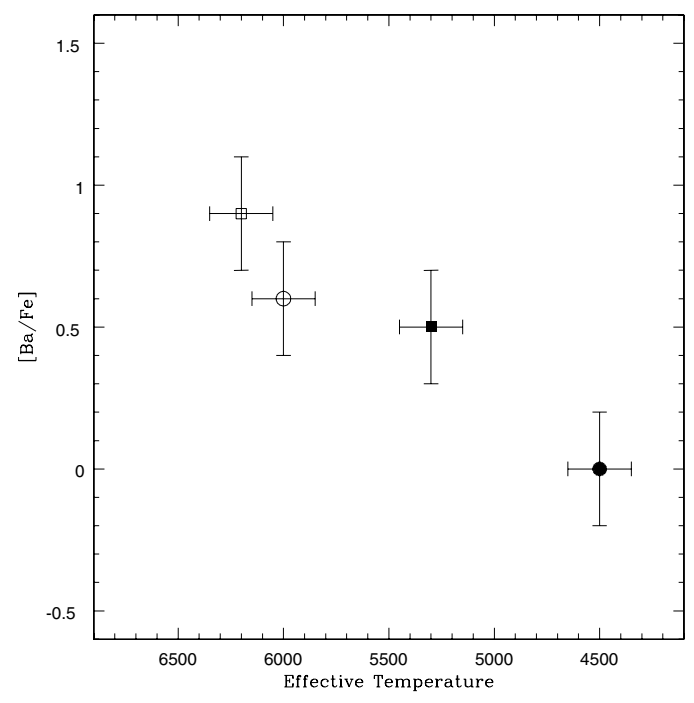

Fig. 7. $[\mathrm{Ba} / \mathrm{Fe}]$ vs. effective temperature for $\mathrm{D}^{\prime}$-type symbiotics and Me 1-1. Symbols have the same meaning as in Fig. 6.

under discussion here consist of subgiants evolving across the so-called "Hertzsprung Gap" to the red giant branch (RGB). Stars of the approximate mass range spanned by these three $\mathrm{D}^{\prime}$-symbiotics $\left(\sim 2-4 M_{\odot}\right)$ have very small, to near-zero mass convective envelopes on, or near the main sequence. As they evolve across the Hertzsprung Gap and transform from core $\mathrm{H}$-burning to shell H-burning around an inert He-core (at the base of the RGB), an increasingly massive convective envelope forms. As a point of reference, a $3 M_{\odot}$ star arrives at the base of the RGB with a convective envelope mass of $\sim 1.9 M_{\odot}$. Thus, any barium enriched material that was transferred onto a $2-4 M_{\odot}$ star while it was near the main sequence or just beginning to evolve onto the subgiant branch, would find the accreted material significantly diluted by the time the star had evolved to the base of the RGB.

The interpretation of the rapidly rotating Ba-enriched $\mathrm{D}^{\prime}$-symbiotic stars is that they result from wind accretion on the currently observed star from its companion, when the companion was a luminous, mass-losing, s-process enriched AGB star. This model was developed by Jeffries \& Stevens (1996) and their modelling suggests that typical accreted masses would be roughly $0.1-0.3 M_{\odot}$. If such a mass of Ba-enriched material were accreted onto the less-evolved star while it was near the main-sequence, this material would subsequently be diluted by roughly a factor of 10 as the star evolved to the base of the RGB. This effect is what is observed in Fig. 7 and strengthens the wind accretion model.

The non-detection of barium enrichment is not the only difference observed between Me 1-1 and the D'-type symbiotic stars. The nebular density of Me $1-1$ is $10^{4} \mathrm{~cm}^{-3}$ (Shen et al. 2004), which is much lower than that found in typical $\mathrm{D}^{\prime}$-type symbiotics of $10^{6}-10^{7} \mathrm{~cm}^{-3}$. Therefore, in the diagram $5007 / \mathrm{H} \beta$ versus $4363 / \mathrm{H} \gamma$ Me 1-1 occupies a different position from the $\mathrm{D}^{\prime}$-type symbiotics. In Fig. 8 we show such a diagram for $\mathrm{PNe}$ and symbiotic stars, where the position of Me 1-1 is indicated with an open circle.

Cross-correlation techniques as those employed to obtain the orbital periods of the $\mathrm{D}^{\prime}$-type symbiotics (Zamanov et al. 2006) would be necessary to provide a better understanding of the formation of such binary systems. 


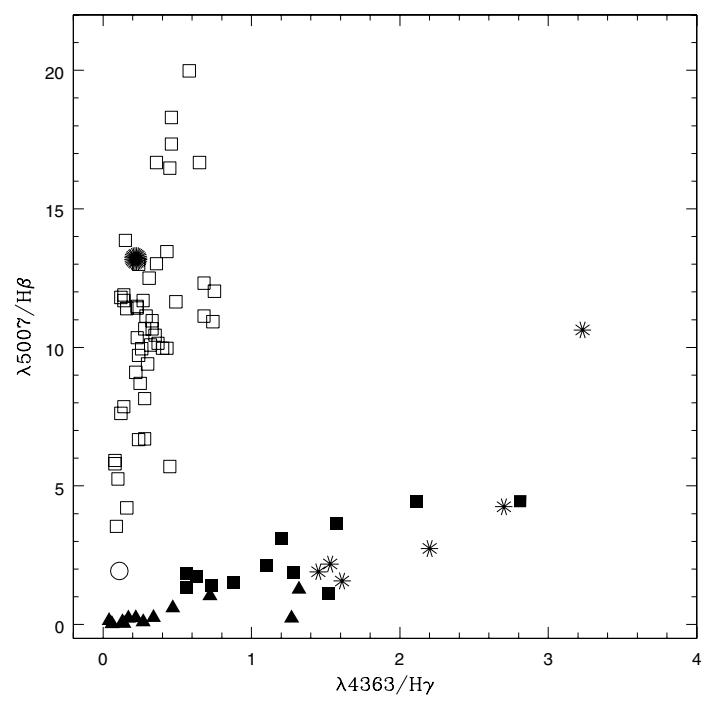

Fig. 8. Position of planetary nebulae, $\mathrm{D}^{\prime}-$, D- and S-type symbiotics, in the $\lambda 5007 / \mathrm{H} \beta-\lambda 4363 / \mathrm{H} \gamma$ plane. Open squares, planetary nebulae; filled square points, D-type symbiotics; filled triangles, S-type symbiotics; $\mathrm{D}^{\prime}$-type symbiotics, asterisks; Me 1-1, open circle. Data from Kingsburgh \& Barlow (1994), Schmid \& Nussbaumer (1993), Gutiérrez-Moreno \& Moreno (1996), Schmid \& Schild (1990), Pereira (1995), Pereira et al. (1998), Gutiérrez-Moreno et al. (1999).

\subsection{The physical structure of Me 1-1 and comparison with other planetary nebulae}

Morphologically, Me 1-1 has been classified as a peculiar or an elliptical PN (Manchado et al. 1996; Stanghellini et al. 2002). However, the direct images strongly suggest that Me 1-1 is a ring nebula seen edge-on. The two outer emission knots trace the edges of the ring while, as already mentioned, the central knot is the central star. The plane of the ring is oriented at PA $352^{\circ}$. The faint "V"-shaped emission emmanating from the outer knots points to the existence of incipient bipolar lobes. Therefore, within the classical morphological classification of PNe (round, elliptical and bipolar), Me 1-1 would fit better into the bipolar class. The small range of expansion velocities $\left(10-13 \mathrm{~km} \mathrm{~s}^{-1}\right)$ suggests that the ring expands like a "solid" structure with a very small internal velocity gradient. Assuming a mean radius of $1.1^{\prime \prime}$ for the ring, a mean expansion velocity of $12 \mathrm{~km} \mathrm{~s}^{-1}$ and the distance of $6 \mathrm{kpc}$ derived above, the kinematical age of Me $1-1$ is $\simeq 2600$ yr.

The morphology of Me 1-1 is very similar to that of other $\mathrm{PNe}$ in which a bright ring/toroid is the dominant nebular structure, usually accompanied by incipient and/or faint bipolar lobes. This is the case of IC 2149, A 79, He 2-428, SuWt 2, WeBo 1 and Sp 1 (Vázquez et al. 1999; Exter et al. 2003; Bond et al. 2003; Bond \& Livio 1990; Rodríguez et al. 2001). It is worth noting that most of these ring-like PNe host binary central stars. In the case of Me 1-1, the central star appears displaced from the nebular center by $\sim 400 \mathrm{AU}$. This displacement is within the range of those found in other PNe (e.g., Soker 1994; and Miranda et al. 2001, references therein). Remarkably, off-center central stars are observed in most ring-like PNe, after visual inspection of their images (see references above). This result provides strong support to models that interprete off-center central stars as a consequence of their binary nature (Soker 1994; Soker et al. 1998). Although the evolutionary status of these ring-like PNe and the binary characteristics of their central stars differ among the different objects, the morphological similarities suggest that these $\mathrm{PNe}$ share a common formation history. In particular, the formation of a ring-like PN requires that the former AGB star in the system ejects virtually all mass towards a plane, probably forming a circumbinary ring or a highly flattened envelope. The presence of a companion appears to be crucial in this processes (see, e.g., Bond \& Livio 1990).

\section{Conclusions}

We have analyzed the absorption and emission spectrum of the peculiar planetary nebula Me 1-1 using high resolution spectroscopy, complemented with direct images. The main conclusions of this work can be summarized as follows:

1. The stellar parameters derived for the cool star in Me 1-1 are: $T_{\text {eff }}=4500 \mathrm{~K}, \log g=2.0$ and $V_{\text {rot }}=90 \mathrm{~km} \mathrm{~s}^{-1}$. These result in a $\mathrm{K}(1-2) \mathrm{II}$ spectral type, a mass of $3.5 M_{\odot}$, a distance of $6 \mathrm{kpc}$ and a luminosity of $370 L_{\odot}$.

2. The cool star presents solar abundances of iron and calcium. Barium, a monitor of s-process nucleosynthesis, does not display any degree of enrichment. One possibility to explain the lack of barium enrichment is that the cool star of Me 1-1 may have reached the base of the RGB and the accreted matter has been diluted by the deepening convective envelope.

3. The direct images strongly suggests that Me 1-1 is a ring-like planetary nebula seen egde-on, with faint, incipient bipolar lobes. The central star is displaced $\sim 400 \mathrm{AU}$ from the center of the nebula, a fact consistent with its binary nature.

4. Expansion velocity of the ring ranges from 10 to $13 \mathrm{~km} \mathrm{~s}^{-1}$ with no dependence on the ionization potential of the specie. Broad wings $\left(\simeq 600 \mathrm{~km} \mathrm{~s}^{-1}\right)$ are detected in the $\mathrm{H} \alpha$ emission line, probably with a stellar origin.

5. A comparison of Me 1-1 with other ring-like planetary nebulae shows that most of them hosts off-center, binary central stars suggesting that a binary nature is a basic ingredient for the formation of ring-like planetary nebulae.

Acknowledgements. We thank our referee for useful comments that have improved the presentation and interpretation of the data. We thank the Calar Alto Observatory for allocation of director's discretionary time to this programme and to the staff for the FOCES spectrum of Me 1-1. We are grateful to M. A. Guerrero for providing us with the narrow-band images of Me 1-1. This paper has been supported partially by MEC grant AYA2005-01495 (co-funded with Feder Funds) and Junta de Andalucía grant FQM1747.

\section{References}

Acker, A., et al. 1992, Strasbourg - ESO catalogue of galactic planetary nebulae Bessell, M. S., Castelli, F., \& Plez, B. 1998, A\&A, 333, 231

Bond, H. E., \& Livio, M. 1990, ApJ, 355, 568

Bond, H. 2000, Binarity of Central Stars of Planetary Nebulae, ed. J. H. Kastner, N. Soker, \& S. Rapapport, in From Origins to Microstructures, ASP Ser., 199, 115

Bond, H. E., Pollacco, D. L., \& Webbink, R. F. 2003, AJ, 125, 260

Cahn, J. H., Kaler, J. B., \& Stanghellini, L. 1992, A\&AS, 94, 399

Exter, K., Bond, H. E., Pollaco, D., \& Dufton, P. 2003, in Planetary Nebulae: Their Evolution and Role in the Universe, ed. S. Kowk, M. Dopita, \& R. Suttherland (ASP), IAU Symp., 209, 234

Gutiérrez-Moreno, A., \& Moreno, H. 1996, PASP, 108, 972

Gutiérrez-Moreno, A., Moreno, H., \& Cortés, G. 1995, PASP, 107, 462 Gutiérrez-Moreno, A., Moreno, H., \& Costa, E. 1999, PASP, 111, 571 Jeffries, R. D., \& Stevens, I. R. 1996, MNRAS, 279, 180

Kingsburgh, R. L., \& Barlow, M. J. 1994, MNRAS, 271, 257 (KB94) Kurucz, R., \& Bell, B. 1995, CD-ROM 23, Atomic Line Data (Cambridge: SAO) Lutz, J. H. 1977, A\&A, 60, 93

Lutz, J. H. 1984, ApJ, 279, 720 
Manchado, A., Guerrero, M. A., Stanghellini, L., \& Serra-Ricart, M. 1996, The IAC morphological catalog of northern Galactic planetary nebulae (Instituto de Astrofísica de Canarias)

Miranda, L. F., Torrelles, J. M., Guerrero, M. A., Vázquez, R., \& Gómez, Y. 2001, MNRAS, 321, 487

Pfeiffer, M. J., et al. 1998, A\&AS, 130, 831

Perek, L., \& Kohoutek, L. 1967, Catalogue of Galactic Planetary Nebulae, Czechoslovak Academy od Sciences, Prague

Pereira, C. B. 1995, A\&AS, 111, 471

Pereira, C. B., Landaberry, S. J. C., \& Junqueira, S. 1998, A\&A, 333, 658

Pereira, C. B., Smith, V. V., \& Cunha, K. 2005, A\&A, 429, 993

Phillips, J. P. 2004, MNRAS, 353, 589

Rodríguez, M., Corradi, R. L. M., \& Mampaso, A. 2001, A\&A, 377, 1042

Schaerer, D., Charbonnel, C., Meynet, G., Maeder, A., \& Schaller, G. 1993 , A\&AS, 102,339
Schmid, H. M., \& Schild, H. 1990, MNRAS, 246, 84

Schmid, H. M., \& Nussbaumer, H. 1993, A\&A, 268, 159

Schmidt-Kaler, T. 1982, in Landolt-Börnstein New Series, ed. K. Schaifers, \& H. H. Vigt, Group 4, Vol. 2b (Berlin: Springer), 449

Shen, Z.-X., Liu, X.-W., \& Danziger, I. J. 2004, A\&A, 422, 563

Smith, V. V., Cunha, K., Jorissen, A., \& Boffin, H. M. J. 1996, A\&A, 315, 179

Smith, V. V., Pereira, C. B., Cunha, K., et al. 2001, ApJ, 556, L55

Sneden, C. 1973, Ph.D. Thesis, Univ. of Texas

Soker, N. 1994, MNRAS, 270, 774

Soker, N., Rappaport, S., \& Harpaz, A. 1998, ApJ, 496, 842

Stanghellini, L., Villaver, E., Manchado, A., \& Guerrero, M. A. 2002, ApJ, 576, 285

Thévenin, F., \& Jasniewicz, G. 1997, A\&A, 320, 913

Vázquez, R., Miranda, L. F., Torrelles, J. M., et al. 2002, ApJ, 576, 860

Zamanov, R. K., Bode, M. F., Melo, C. H. F., et al. 2006, MNRAS, 365, 1215 